\title{
Automatic Determination of Blood Flow Velocity in Brain Microvessels in a Cerebral Infarction Model Mouse Using a Small Implantable CMOS Imaging Device
}

\author{
Tetsuo Sato,,${ }^{* * *, \#}$ Haruka Dejima, ${ }^{* *}$ Makito Haruta, ${ }^{* *}$ Satoshi Kamikawa, ${ }^{* * *}$ Hitomi NakaZawa, ${ }^{\dagger}$ Takashi Tokuda, ${ }^{* *}$ \\ Jun OHta, ${ }^{* *}$ Shigehiko KanaYA ${ }^{* *}$
}

\begin{abstract}
In this study, we used a small implantable complementary metal-oxide-semiconductor (CMOS) imaging device developed by our research group to estimate the blood flow changes by focusing on the movement of red blood cells in the captured movements. Conventional methods for determining blood flow velocity have limitations in that variation occurs due to manual measurement. Therefore, we developed a novel technique to measure blood flow in the brain. This method involves calculating the changes of selected pixels using a normalized cross-correlation coefficient. Using this cross-correlation method, we analyzed a mouse cerebral infarction model to detect changes in brain activity. The results of analysis showed that the average velocity of blood flow upstream of the infarction site decreased while the velocity in blood vessel parallel to the infarction increased after occlusion was induced. These results thus confirmed that the new method can detect blood velocity changes, suggesting the feasibility of the cross-correlation method for estimating blood flow velocity.
\end{abstract}

Keywords: blood flow velocity, brain microvessel, CMOS imaging device, automatic determination.

Adv Biomed Eng. 6: pp. 68-75, 2017.

\section{Introduction}

\subsection{Background}

In the 1890s, Roy and Sherrington [1] revealed that an increase in local cerebral blood flow is associated with the activity of nerve cells in the brain. Upon exercise and sensory stimulation, nerve cells in the brain become excited, and substantial energy is required for the synthesis of potential changes and neurotransmitters. To produce energy for nerve cells, the metabolic activity of glycolysis involving oxygen occurs, which generates ATP. However, oxygen and glucose are rarely stored in the brain. Thus, for metabolic activity, oxygen bound to hemoglobin in red blood cells along with glucose are transported via the blood to the brain to maintain brain homeostasis. This process requires an increase of cerebral blood flow [2]. Since this mechanism was discovered, substantial efforts have been made to clarify how blood flow is regulated in the brain.

Many methods including functional magnetic resonance imaging, positron tomography, and noninvasive near-infrared spectroscopy have been used to measure blood flow changes in the brain cortex $[3,4]$. These diagnostic techniques are based on the amount of blood per unit volume of brain tissue, referred to as cerebral blood volume; and the blood flow in one minute per unit

This study was presented at the Symposium on Biomedical Engineering 2016, Asahikawa, September, 2016.

Received on July 22, 2016; revised on October 30, 2016, January

23, 2017, and April 24, 2017; accepted on May 1, 2017.

* Gunma Prefectural College of Health Sciences, Maebashi, Japan.

** Nara Institute of Science and Technology, Ikoma, Japan.

**** Doshisha University, Kyoto, Japan.

${ }^{\dagger}$ Asahikawa Medical University, Asahikawa, Japan.

\#323-1 Kamioki, Maebashi, Gunma 371-0052, Japan.

E-mail: satote@gchs.ac.jp volume of brain tissue, referred to as cerebral blood flow. However, the spatial resolution of the above methods is approximately 1 to $100 \mathrm{~mm}$. Therefore, the information obtained represents the average blood flow of many blood vessels. The above methods are thus not suitable for estimating blood flow changes at microvascular level, that is, in the order of approximately $10 \mu \mathrm{m}$.

With this background, a two-photon microscope and cerebral blood flow imaging at microvascular level using a custom-made device have been developed using experimental animals, and basic investigations of hemodynamics have been performed. However, since the experimental set-up required is very large, it is necessary to immobilize experimental animals such as mice and rats, thus impeding measurement of changes of brain activity in a free-moving state.

In most brain function measurements, since experimental animals are anesthetized, it is impossible to measure in the waking state. In addition, the spatial resolution is in the range of $1-20 \mathrm{~mm}$, which is too large to perform investigations of physiological functions including blood flow dynamics at microvascular level (blood vessel diameter is approximately $10 \mu \mathrm{m}$ ). Therefore, small brain imaging devices for experimental animals have been developed. Kleinfeld et al. [5] measured brain function using a two-photon microscope. This approach requires a craniotomy and fixing a metal frame to the skull of the experimental animal under anesthesia, making it difficult to perform measurement in freely moving experimental animals. Takuwa et al. [6] measured the brain function of experimental animals in a conscious state using a laser Doppler blood flow meter. The use of this experimental device does not require anesthesia and allows measurement of cerebral blood flow changes, but this method still requires immobilization of the head and only allows free movement of the limbs. Thus, completely free movement of the experimental animals cannot be realized.

In this study, a complementary metal-oxide-semiconductor (CMOS) imaging device was developed by our research group. 
This device is ultra-compact and lightweight, and an LED light source is used to illuminate the brain surface of experimental animals. It is implanted into the head to determine the cerebral blood flow. The use of this device allows imaging of cerebral blood flow in free-moving animals. The wavelength of the LED used is $535 \mathrm{~nm}$, because light of this wavelength is absorbed by hemoglobin in red blood cells. Therefore, the movement of red blood cells can be visualized without injecting a fluorescent dye. Because there is no need to use a fluorescent dye, long-term observation becomes possible.

\subsection{Purpose of the study}

Our CMOS imaging device can be used to determine blood flow velocity using images obtained by the line scan method. However, in this approach, variations arise due to the subjective nature of visual inspection, which may lead to errors in the results obtained. Therefore, the velocity of red blood cells in an image should be automatically calculated using image processing. The purpose of our study was to estimate blood flow changes in images acquired by our CMOS imaging device using cross-correlation method and to verify the feasibility of this method.

This paper is arranged as follows. In section 2, materials and methods are presented. The principle of our measuring device in the present paper is also described. In section 3, blood flow measurement under anesthesia in a mouse model of brain infarction is described. In section 4, discussions are described. Finally, in section 5 , conclusions of this study are presented.

\section{Materials and methods}

\subsection{Materials}

The standard CMOS process $(0.35-\mu \mathrm{m}$ 2-poly 4-metal CMOS; Austria Microsystems) was used in this study. Sensor input and output signals are transferred to a control board through a relay base. This control board is also used for signal transfer to a PC, and the analog signal is converted into a 14-bit digital signal. The device uses nine green LEDs (Epistar, $\lambda=535 \mathrm{~nm}$ ) as light source. The polyimide substrate has a structure of nine green LEDs arranging around a CMOS imaging sensor. The device weighs $0.02 \mathrm{~g}$, which is only one-ten thousandth of the weight of adult rats used as experimental animals (body weight approximately $200 \mathrm{~g}$ ), and measures $3.3 \times 5.3 \times 0.35 \mathrm{~mm}^{3}$, which is ultra-compact and lightweight. This device is associated with a light source implanted into the head to illuminate the brain surface of the experimental animal, and has a direct contact element to acquire images. The device has no objective lens and is small, lightweight, and allows imaging of cerebral blood flow in a freely moving state without head immobilization [7].

Light emitted by the light source scatters in the brain. The wavelength of the LED light source is $535 \mathrm{~nm}$, and the light absorbed by hemoglobin in erythrocytes flowing through the blood vessels appears dark in the images obtained. There is no need to use a fluorescent dye, which allows long-term observation. The frame rate of the device is $58 \mathrm{~Hz}$, the pixel size of the sensor is $7.5 \times 7.5 \mu \mathrm{m}^{2}$, and the pixel array has $120 \times 268$ pixels.

In this study, focal cerebral ischemia was induced artificially in mice to create cerebral infarction models. C57BL/6J mice (SLC, ten weeks of age, male) were used. The cerebral blood flow in the ischemic site, upper portion of the ischemic site, and a branch in the upper portion were observed. All animal procedures conformed to the animal care and experimentation guidelines of Nara Institute of Science and Technology.

First, each mouse was placed under urethane anesthesia and the scalp and skull were removed for observation. Then, a photothrombosis method was used to induce local cerebral ischemia. Rose Bengal solution ( $1 \mathrm{ml} / \mathrm{kg}$ ), a light-receiving dye, was injected into the tail vein. A thrombus was produced in a brain surface vessel by laser irradiation using a laser light source and optical fiber with wavelength of $532 \mathrm{~nm}$. The conditions of laser irradiation were as follows: light intensity of $0.2 \mathrm{~mW}$ for approximately $1 \mathrm{~min}$ within a range of $0.2 \mathrm{~mm}$ [8]. Images were obtained before cerebral infarction (early stage), during the creation of focal cerebral ischemia (middle stage), and after cerebral infarction (late stage).

To investigate the relationship between focal cerebral ischemia and blood flow, a single-vessel ischemic state was tested. The irradiation site is shown by a yellow circle in Fig. 1a. During and after laser irradiation, the blood flow was stopped (Fig. 1b and c). In the experiment, the CMOS imaging device was positioned at the site of light irradiation and blood flow was measured.

To automatically estimate the blood flow velocity by the change in intensity of the selected pixel, the normalized cross-correlation method was used. Nine portions of the blood vessel were selected, as shown in Fig. 1d.

\subsection{Methods}

\subsubsection{Conventional manual analysis}

Blood flow velocity is conventionally analyzed by the line scan method. First, an average image in a frame interval is obtained. Then, a 'difference image' representing the difference between two images taken at the respective intervals is produced. Since there are few changes other than changes in the red blood cells, it is possible to capture the red blood cells using this difference image. Next, a pixel representing the blood vessel position is selected within the difference image. Subsequently, a 'stack image' is prepared, arranged in a vertical direction with a continuous frame. Since the red blood cells are darker areas than other areas within the image, the slope of darker areas in the stack image is inversely proportional to the rate at which red blood cells are moving.

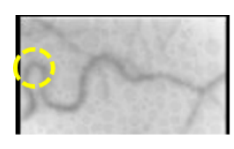

(a) early

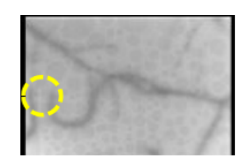

(b) middle

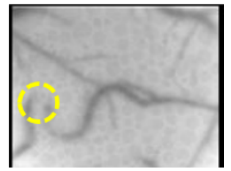

(c)

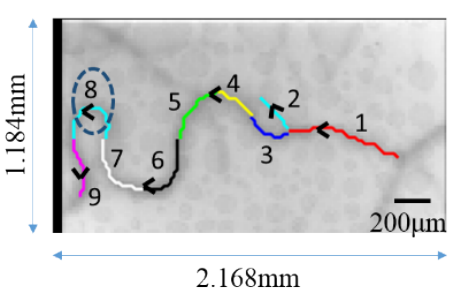

(d)

Fig. 1 Brain infarction images. (a) Before laser irradiation, (b) during irradiation, (c) after irradiation, and (d) nine portions of the blood vessel including the infarction site. 
The spatial resolution of the device is $7.5 \mu \mathrm{m}$ and time resolution is $1 / 58 \mathrm{~s}$. Red blood cells of Sprague-Dawley rats have a diameter of approximately $8 \mu \mathrm{m}$ [9]. The spatial resolution of the experimental device is thus insufficient to observe each erythrocyte, but groups of erythrocytes moving in a vessel can be used to assess blood flow. Blood vessel diameters of 14 to $35 \mu \mathrm{m}$ were selected for further velocity measurements.

Equation (1) shows how the blood flow velocity is determined by the line scan method. The duration of movement of red blood cells is dt and the movement distance is ds [10].

$$
V=\frac{d s}{d t}
$$

However, the method of determining the slopes caused by red blood cells described so far involves visual inspection, which is associated with the problem of variation due to subjectivity of the assessment method. Therefore, the velocity of the red blood cells in the image should be automatically determined quantitatively using image processing. We propose a method for automatic calculation of the velocity using correlation coefficient.

\subsubsection{Velocity estimation method using correlation coeffi- cient}

In this section, we propose a method to estimate the velocity of blood flow from the selected pixel using the normalized cross-correlation function [11]. The procedure that consists of six steps is referred to as the cross-correlation method. The advantages of this procedure are that it can be used in real time during the experiment and can be performed automatically without human involvement. The average image in the frame interval $N$ is used to create a difference image.

\section{Step1.}

$P$ pixels representing the position of the blood vessel in the difference image are selected (Fig. 2; step1).

\section{Step2.}

A Sobel filter is used to find an edge. The pixel is then stacked in the vertical direction (Fig. 2; step2). Vertical edges are noises. To emphasize the horizontal edges, a kernel size of $3 \times 3$ pixels of the Sobel filter is selected to reduce the vertical edges.

$$
S=\left[\begin{array}{ccc}
1 & 2 & 1 \\
0 & 0 & 0 \\
-1 & -2 & -1
\end{array}\right]
$$

\section{Step3.}

The selected frame section for estimating the velocity is $L$, and the selected pixel number is $P$. The intensity change is then determined between frames for each pixel. The pixel located in the center within the selected pixel is used as a reference pixel. The frame section $L$ is set at $2 P$ so that the edges reach both sides of the image of the selected pixel number $P$ (Fig. 2 step3).

\section{Step4.}

The normalized cross-correlation of the selected pixel Y and the reference pixel $\mathrm{X}$ is calculated. $\mathrm{Y}$ is shifted one frame at a time to calculate the degree of similarity.

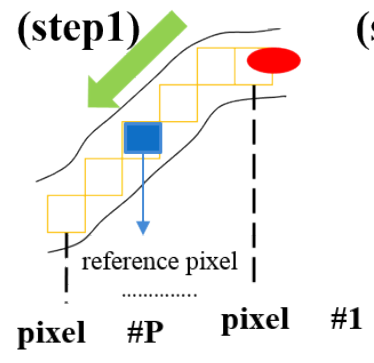

(step2)
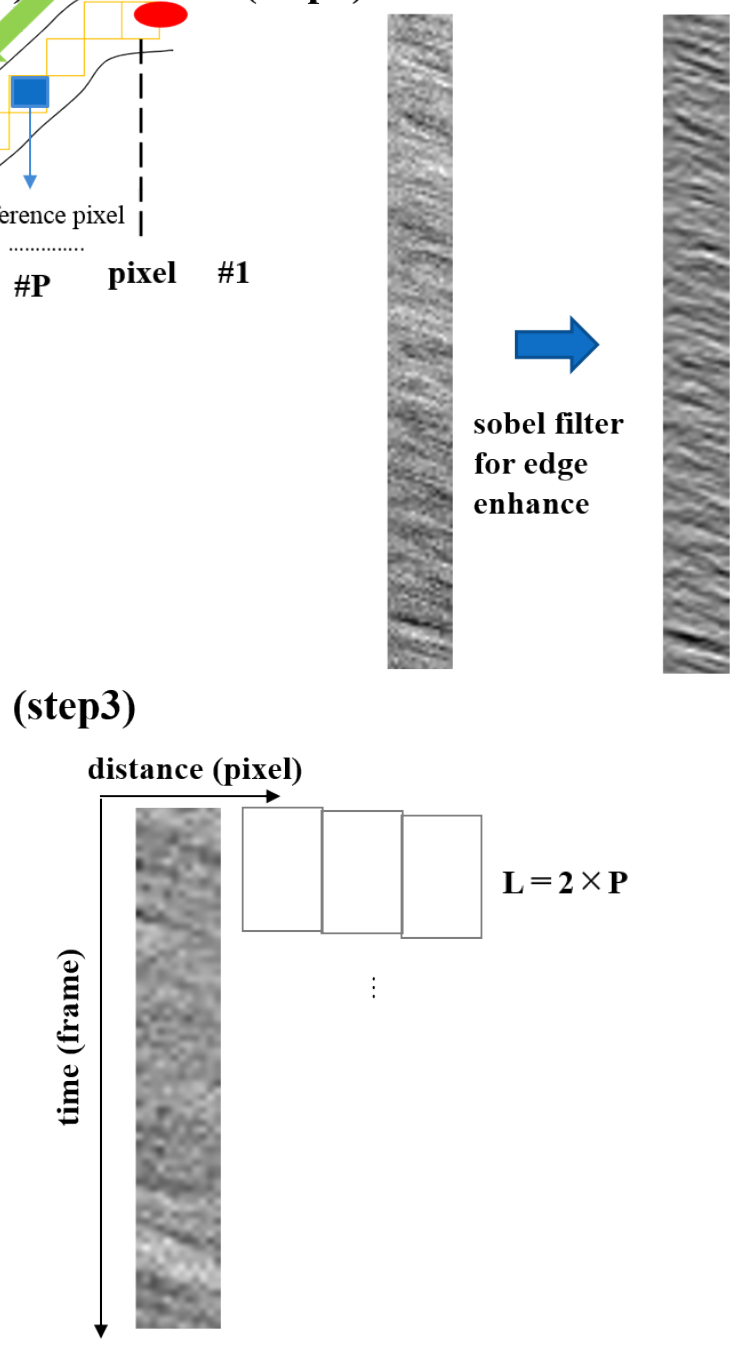

Fig. 2 Schematic drawing of the proposed method. (step1) Selecting the pixel along the blood vessel, (step2) applying the Sobel filter, (step3) selecting the frame section.

$$
\begin{array}{r}
R_{X Y(\tau)}=\frac{\sum_{i=1}^{L}\left(x_{i}-\bar{x}\right)\left(y_{i+\tau}-\bar{y}\right)}{\sqrt{\sum_{i=1}^{L}\left(x_{i}-\bar{x}\right)^{2}} \sqrt{\sum_{i=1}^{L}\left(y_{i}-\bar{y}\right)^{2}}} \\
(\tau=-L+1,-L+2, \ldots L-1)
\end{array}
$$

Here, $x_{i}$ is the pixel intensity of the reference, $\bar{x}$ is the average intensity of the reference, $y_{i}$ is the pixel intensity of each selected pixel, $\bar{y}$ is the average of each pixel, and $\tau$ is the frame difference. If the normalized cross-correlation is the maximum, and only greater than or equal to a threshold value, the distance and time difference between the selected pixel and the reference was plotted. The time difference is calculated from the frame rate. The threshold is set to 0.6. This was determined empirically from the error rate of manual measurement [11] (Fig. 3; step4).

\section{Step5.}

A regression line from the scatter diagram is obtained using the least squares method. The slope represents the blood flow velocity (Fig. 3; step5). 
(step4)

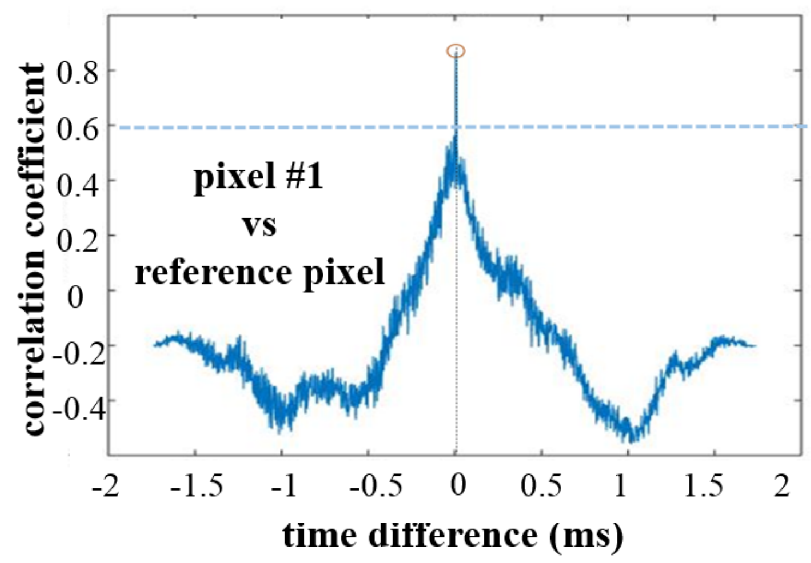

(step5)

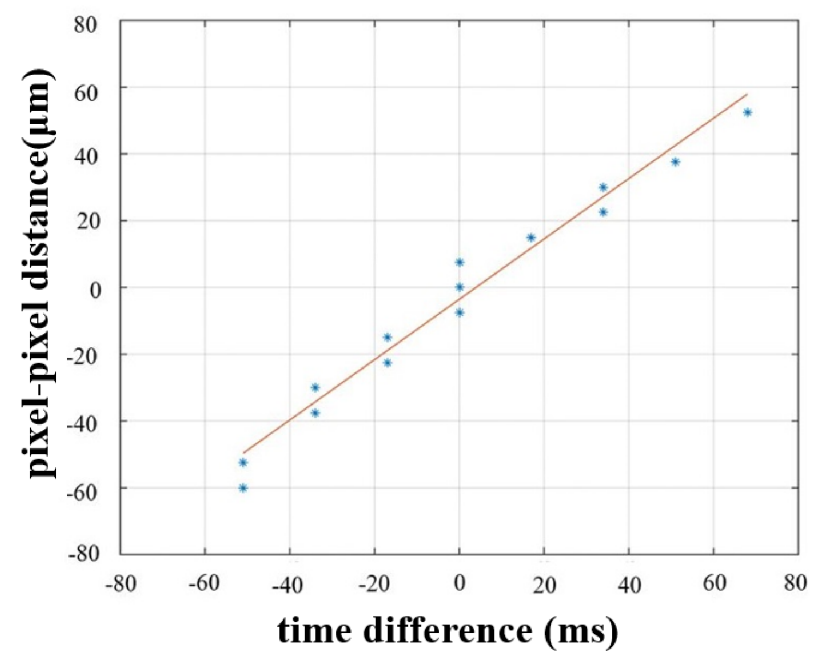

(step6)

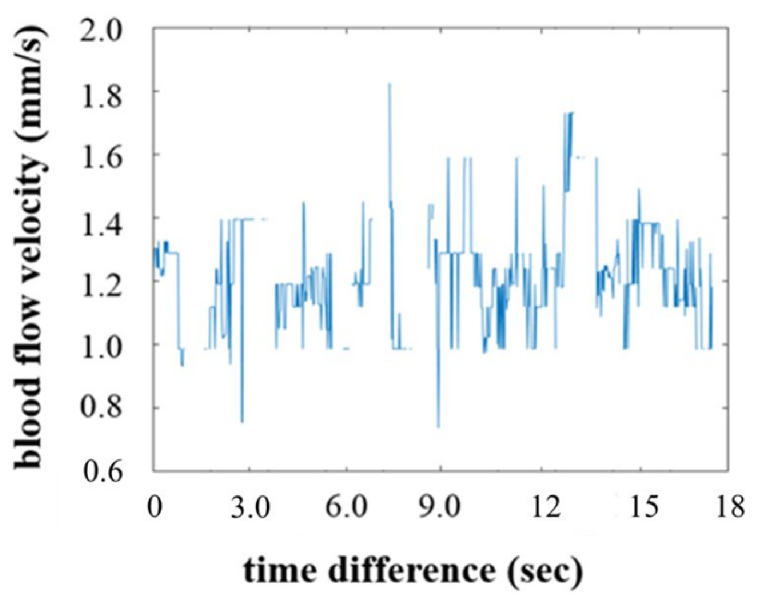

Fig. 3 Schematic drawing of the proposed method. (step4) Calculating the correlation coefficient between the pixel, (step5) plotting the relationship between the time and the distance, and (step6) calculating the blood flow velocity.

\section{Step6.}

Frame $L$ is then be shifted by one frame. Steps 2 to 5 are then e repeated. Eventually, a time series of blood flow velocity is obtained (Fig. 3; step6).

The sampling numbers used for calculating blood flow ve-
Table 1 Sampling numbers for calculating blood flow velocity.

\begin{tabular}{lllllll}
\hline & \multicolumn{5}{c}{ sampling number } \\
\cline { 2 - 7 } stages & early & \multicolumn{3}{c}{ middle } & late \\
\hline methods & $\begin{array}{c}\text { manual } \\
\text { proposed } \\
\text { method }\end{array}$ & $\begin{array}{l}\text { manual } \\
\text { proposed } \\
\text { method }\end{array}$ & $\begin{array}{l}\text { manual } \\
\text { proposed } \\
\text { method }\end{array}$ \\
\hline ROI 1 & 17 & 417 & 26 & 464 & 14 & 803 \\
ROI 2 & 15 & 452 & 16 & 498 & 24 & 900 \\
ROI 3 & 31 & 545 & 25 & 560 & N/D & N/D \\
ROI 4 & 27 & 649 & 16 & 343 & N/D & N/D \\
ROI 5 & 19 & 491 & 21 & 88 & N/D & N/D \\
ROI 6 & 26 & 588 & 10 & 139 & N/D & N/D \\
ROI 7 & 26 & 789 & 12 & 240 & N/D & N/D \\
ROI 9 & 19 & 669 & 12 & 70 & N/D & N/D \\
\hline
\end{tabular}

$\mathrm{N} / \mathrm{D}$, not determined.

locity by manual and correlation coefficient analyses are shown in Table 1.

\section{Results}

Cerebral infarction, in which blood from the arteries is cut off and the oxygen supply to brain tissue becomes insufficient, results in necrosis of nerve cells in the brain. The recovery of brain function from stroke is particularly important and is also related to the recovery of several body functions. To understand the process of recovery of brain function, it is necessary to measure brain function continuously at the same site during the recovery process. Our experimental device allows continuous cerebral blood flow measurement in freely moving animals.

\subsection{Comparison of manual procedure and cross-correlation method}

Using the correlation method, a decrease in blood flow of approximately $15 \%$ was observed $(p<0.01)$ upstream of the ischemic site between the middle and late stages of infarction (Fig. 4; ROI 1). Meanwhile, in the blood vessel parallel to the infarcted vessel, an increase in blood velocity of approximately $60 \%$ was observed between the early and middle stages $(p<0.01)$ (Fig. 4; ROI 2). In addition, decreases in blood flow velocity were seen at ROIs 3, 4, 5 and 7 in the middle stage of infarction $(p<0.01)$ (Fig. 5; ROIs 3-5, Fig. 6; ROI 7). At ROI 8, which was the infarction site, measurement of the blood flow velocity was not possible in the middle and late infarction stages because of the cessation of blood flow, and no results are shown in Figs. 4-6. At ROIs 3 to 9, which were directly connected to the infarction site, measurement of velocity was also not possible in the late stage of infarction, and again no results are shown in Figs. 4-6. In these cases, the correlation coefficients did not exceed 0.6 and the velocity was not calculated because of the unreliability. In our experiment, the minimum blood flow velocity was $0.35 \mathrm{~mm} / \mathrm{s}$ and our methodological minimum was $0.22 \mathrm{~mm} / \mathrm{s}$. At ROIs 6 and 9 , the blood flow velocity had a different tendency compared to the adjacent region due to large measurement errors.

By manual measurement, there was an increase in blood flow velocity of approximately $30 \%$ between the early and middle stages of infarction, and a decrease in blood flow velocity of ap- 

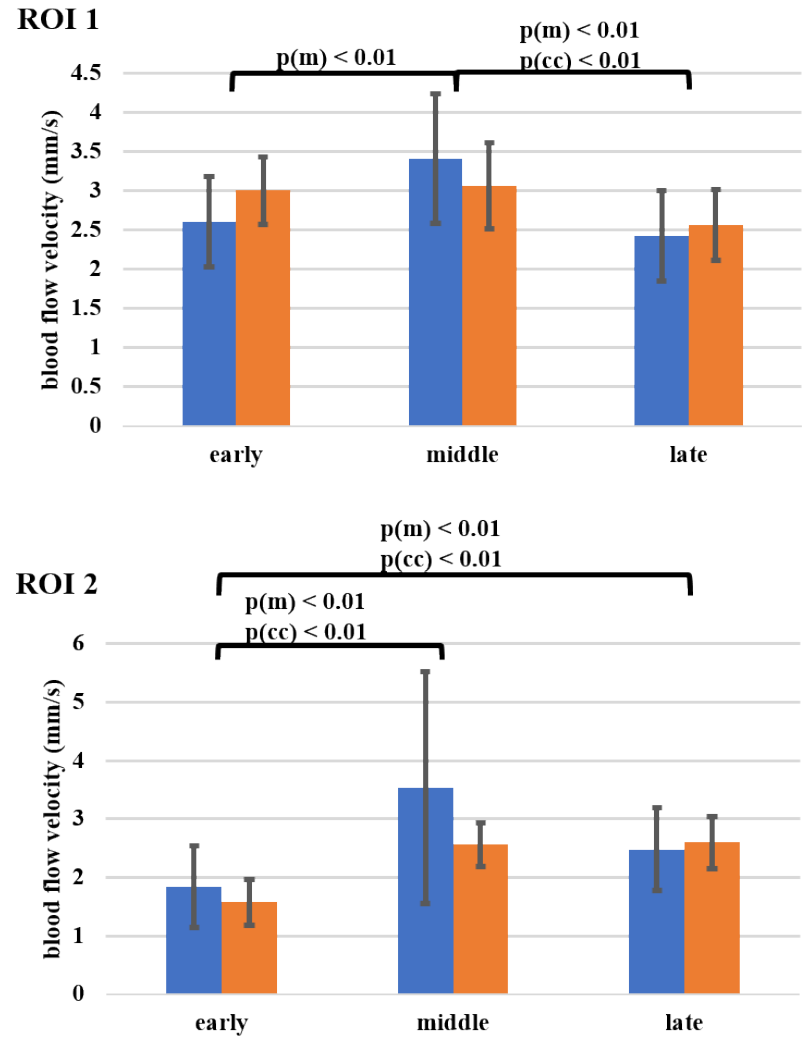

Fig. 4 Blood flow velocity obtained by the manual and correlation coefficient-based methods for ROIs 1 and 2. Blue bar corresponds to the manual method and orange the correlation method. $\mathrm{p}(\mathrm{m})$ and $\mathrm{p}(\mathrm{cc})$ represent the $\mathrm{p}$ values between two conditions, such as early, middle, and late stages, for the manual method and the correlation method, respectively.

proximately $30 \%$ between the middle and late stages $(p<0.01)$ upstream of the ischemic site (Fig. 4; ROI 1). In the blood vessel parallel to the infarcted vessel, an increase in blood flow velocity of approximately $90 \%$ was found between the early and middle stages of infarction $(p<0.01)$ (Fig. 4; ROI 2). Furthermore, decreases in blood flow velocity between the early and middle stages were observed at ROIs 3 to $9(p<0.01)$ (Fig. 5; ROIs 3-6, Fig. 6; ROIs 7 and 9). Both the manual measurement and the correlation method confirmed a reduction in blood flow velocity at ROI 1 between the middle and late infarction stages. At the blood vessel parallel to the infarct site (ROI 2), an increase in blood flow velocity was also confirmed $(p<0.01)$.

Figure 7 shows a plot of the results of manual measurement ( $V_{m}$ on the horizontal axis) against those of the correlation method ( $V_{c}$, longitudinal axis). A regression line with a slope of 0.7992 and a determination coefficient of 0.9142 were obtained. The slope of the correlation method in a cerebral infarction model can be corrected using these data.

\section{Discussion}

In each vessel, the effect of local cerebral ischemia on blood flow was similar to the findings in a previous study reported by Schaffer et al. [12]. Upstream of the infarction, the velocity of blood flow decreased by approximately $15 \%$ from the early stage to the late stage post-infarction. Schaffer et al. reported that the rate of reduction was approximately $60 \%$. Although these two values dif-
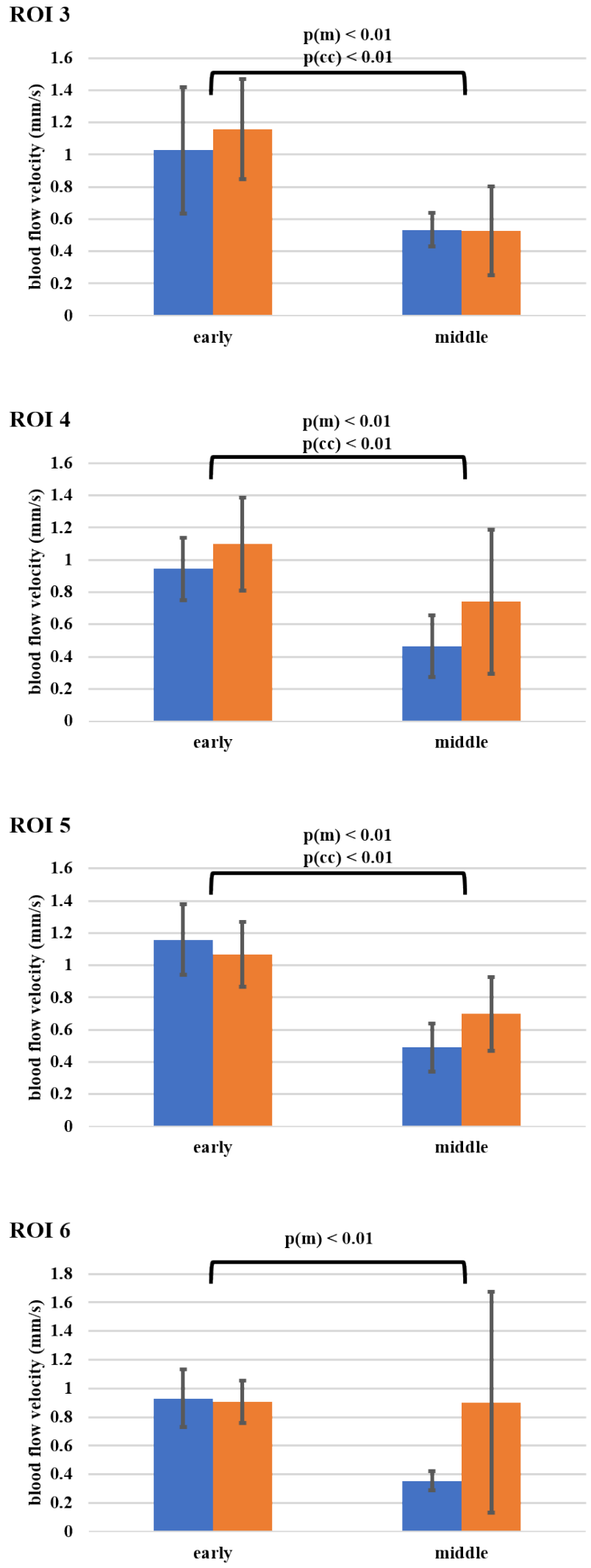

Fig. 5 Blood flow velocity obtained by the manual and correlation coefficient-based methods for ROIs 3-6.

fer, they both confirm decreases. In the vessels located parallel to the cerebral infarction, there was an equivalent increase of approximately $60 \%$, while Schaffer et al. reported an increase of 


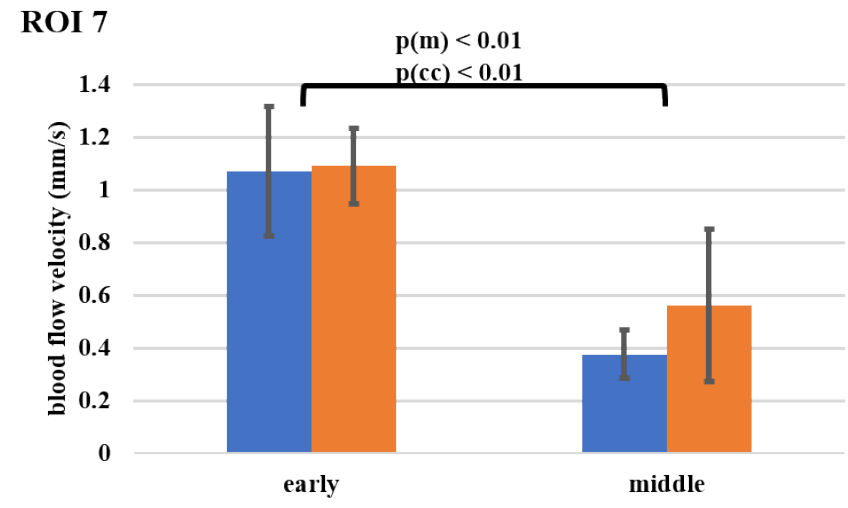

\section{ROI 9}

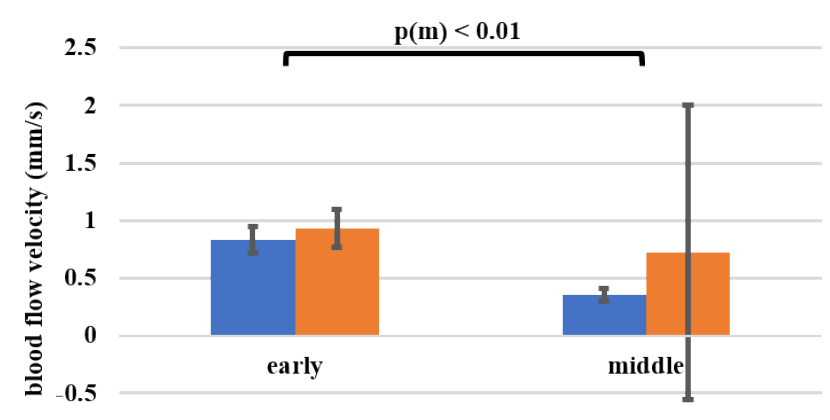

$-1$

Fig. 6 Blood flow velocity obtained by the manual and correlation coefficient-based methods for ROIs 7 and 9 .

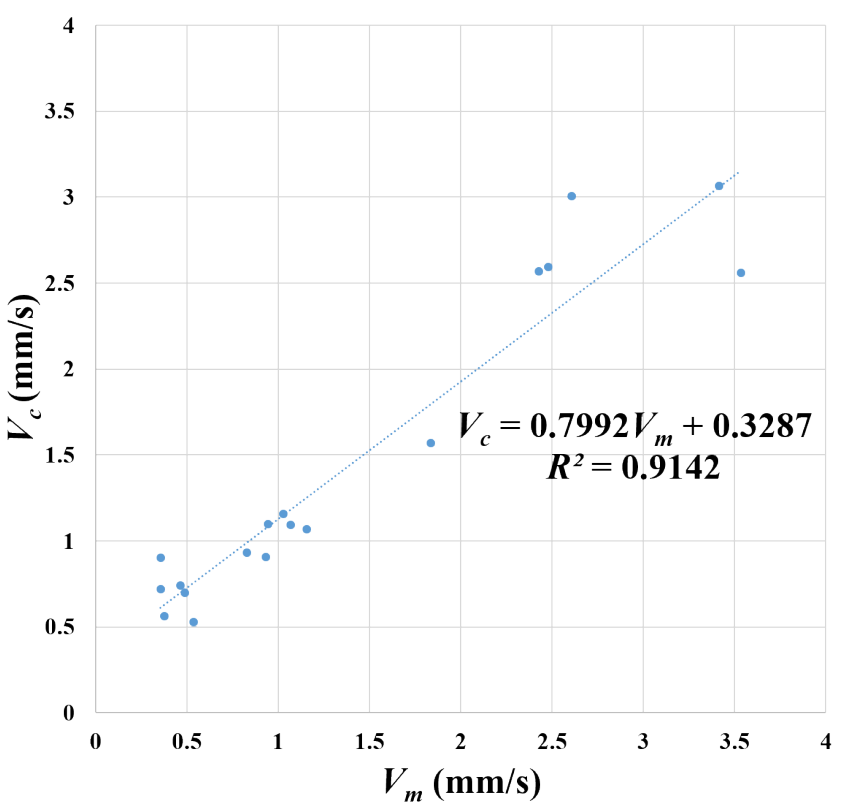

Fig. 7 A scatter plot of the relationship between the blood flow velocity obtained by manual method $\left(V_{m}\right)$ and that obtained by the correlation method $\left(V_{c}\right)$.

about $17 \%$. Although these rates are again different, increases are confirmed in both studies. Furthermore, in the blood vessels in the infarcted area, a decrease in blood flow velocity was observed from the middle to the late stage of infarction. The result of this decrease is an increase in blood flow in the vessel parallel to the infarction. This similar tendency of the up/down-stream of the infarction area showed that geometric configuration of blood vessel does not influence the measured value.

Errors in the correlation method include those associated with an increase in noise or difficulty in detecting the line in the stack image. Errors may also arise if the intensity changes within the selected frame interval, or if there is also a partial edge, because it is incorrectly assumed that the velocity of the whole period is measured. In this study, the blood flow velocity determined by the correlation method was higher than that by manual measurement. The correlation method uses the maximum normalized cross-correlation coefficients, only those greater than or equal to a threshold. The method then involves plotting distance and time difference between pixels, generating a regression line by the least-squares method for a scatter diagram, which represents the blood flow velocity. By using only the maximum coefficient of the regression line, the error can be reduced. Furthermore, the blood flow velocity obtained when the number of plots of two or more points are found in the correlation method. The accuracy can be improved if the number of plots is limited to those larger than the threshold.

An advantage of the correlation coefficient method over the manual method is the absence of the problem of variation caused by subjectivity of assessment associated with visual inspection. However, we verified the accuracy of our method by comparing with the manual method, and not with true values. This is a limitation of our study. Further study will be conducted to validate our method by comparing with another method of measuring blood flow velocity, or estimating the accuracy of the manual method.

\section{Conclusion}

In this study, we developed a method for quantitative estimation of blood flow changes using images acquired by a CMOS imaging device. We also used the proposed method to analyze a mouse model of cerebral infarction. Future challenges in this field include the establishment of a method for automatic selection of blood vessels. Currently, we determine the center of the vessel visually on an image and then select pixels one by one, which is a limitation. Another future goal is to develop a system that allows detection of blood flow velocity in real time.

\section{Conflicts of Interest}

The authors declare no conflicts of interest relationship with any companies or commercial organizations based on the definition of Japanese Society of Medical and Biological Engineering.

\section{References}

1. Roy CS, Sherrington CS: On the regulation of the blood-supply of the brain. J Physiol. 11(1-2), pp. 85-108, 1890.

2. Raichle ME, Mintun MA: Brain work and brain imaging. Annu Rev Neurosci. 29, pp. 449-476, 2006.

3. Binkofski F, Buccino G, Posse S, Seitz RJ, Rizzolatti G, Freund $\mathrm{H}$ : A fronto-parietal circuit for object manipulation in man: evidence from an fMRI-study. Eur J Neurosci. 11(9), pp. 32763286, 1999.

4. Miyai I, Tanabe HC, Sase I, Eda H, Oda I, Konishi I, Tsunazawa 
Y, Suzuki T, Yanagida T, Kubota K: Cortical mapping of gait in humans: a near-infrared spectroscopic topography study. Neuroimage. 14(5), pp. 1186-1192, 2001.

5. Kleinfeld D, Mitra PP, Helmchen F, Denk W: Fluctuations and stimulus-induced changes in blood flow observed in individual capillaries in layers 2 through 4 of rat neocortex. Proc Natl Acad Sci U S A. 95(26), pp. 15741-15746, 1998.

6. Takuwa H, Autio J, Nakayama H, Matsuura T, Obata T, Okada E, Masamoto K, Kanno I: Reproducibility and variance of a stimulation-induced hemodynamic response in barrel cortex of awake behaving mice. Brain Res. 1369(19), pp. 103-111, 2011.

7. Haruta M, Kitsumoto C, Sunaga Y, Takehara H, Noda T, Sasagawa K, Tokuda T, and Ohta J: An implantable CMOS device for blood-flow imaging during experiments on freely moving rats. Jpn J Soc Appl Phys. 53(4S), 2014.

8. Shih AY, Nishimura N, Nguyen J, Friedman B, Lyden PD, Schaffer CB, Kleinfeld D: Optically induced occlusion of single blood vessels in rodent neocortex. Cold Spring Harb Protoc. 12, pp. 1153-1160, 2013.

9. Engström KG, Ohlsson L: Morphology and filterability of red blood cells in neonatal and adult rats. Pediatr Res. 27(3), pp. 220226, 1990.

10. Villringer A, Them A, Lindauer U, Einhäupl K, Dirnagl U: Capillary perfusion of the rat brain cortex an in vivo confocal microscopy study. Circ Res. 75(1), pp. 53-62, 1994.

11. Dejima H, Sato T, Haruta M, Nakazawa H, Tokuda T, Ohta J, Kanaya S: Automatic determination of blood flow velocity in brain microvessel of rat using a small implantable CMOS imaging device. Biosensors and Bio-Micro/Nano Technologies, Poster sessions, 3.LB1 Bioinstrumentation, IEEE EMBC, 2015.

12. Schaffer CB, Friedman B, Nishimura N, Schroeder LF, Tsai PS, Ebner FF, Lyden PD, Kleinfeld D: Two-photon imaging of cortical surface microvessels reveals a robust redistribution in blood flow after vascular occlusion. PLoS Biol. 4(2), pp. 258-270, 2006.

\section{Tetsuo SATo}

Tetsuo SATO received Ph.D degree in engineering from Nara Institute of Science and Technology in 2001. He was a research fellow of Fukui Medical University (2001-2002), research fellow of Japan Science and Technology Agency (2002-2003). He was an Assistant Professor at Nara Institute of Sci-

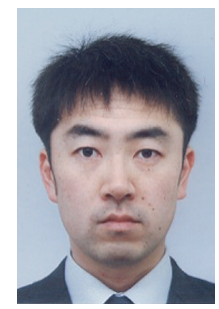
ence and Technology (2003-2016). He is an Associate Professor at Gunma Prefectural College of Health Sciences since 2016. His current research interests are medical image processing, biomedical engineering.

\section{Haruka DEJIMA}

Haruka DejIMA received M.Eng. from Nara Institute of Science and Technology in 2016. Her research interest is biomedical engineering.

\section{Makito HaruTA}

Makito Haruta received Dr. Eng. in material science from NAIST in 2014. In 2016, he joined Graduate School of Materials Science, Nara Institute of Science and Technology, as an assistant professor. His research interest is brain imaging devices for understanding brain functions related to animal behaviors.

\section{Satoshi Kamikawa}

Satoshi Kamikawa is a student of the faculty of life and medical sciences at Doshisha University (2014-). His current research interests are medical image processing, automatic control.

\section{Hitomi NaKazawa}

Hitomi NaKaZawa received M.S. and Dr. Biological Science degrees from the Nara Institute of Science and Technology (NAIST), Nara, Japan, in 2009 and 2012, respectively. She was a research fellow of Osaka University (2012-2014), an Assistant Professor of NAIST (2014-2016), and a re-
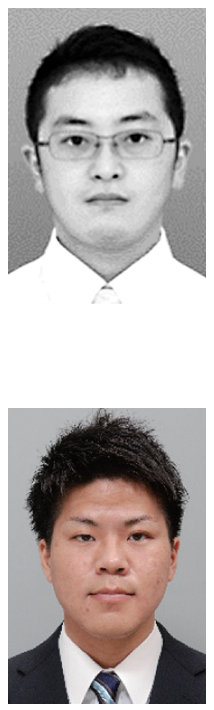
search fellow of Osaka Yukioka College of Health Science (2016 Apr.2016 June). She has been an Assistant Professor at Asahikawa Medical University since 2016 July. Her current research interests are learning and memory, synaptic plasticity, molecular imaging, cell culture, and animal behavioral monitoring. Dr. NAKAZAWA is a member of the Japan Neuroscience Society, the Japanese Society for Neurochemistry, the Japanese Association of Anatomists.

\section{Takashi ToKUDA}

Takashi ToKUDA received his Ph. D. in Materials Engineering from Kyoto University. He is currently working as an associate professor in Graduate School of Materials Science, Nara Institute of Science and Technology, Japan. His research interests include CMOS-based image sensors, bioimaging sensors, and biosensing devices.

\section{Jun ОнтА}

Jun Ohta received his B.E., M.E., and Dr. Eng. degrees in Applied Physics from the University of Tokyo, Japan. In 1983, he joined Mitsubishi Electric Corporation, Hyogo, Japan. In 1998, he joined Graduate School of Materials Science, Nara Institute of Science and Technology, Japan where he is

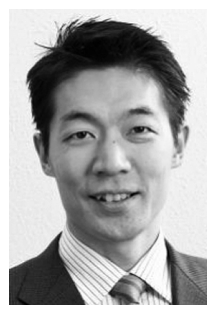
currently working as a professor. His current research interests include retinal prosthesis, and CMOS-based biomedical devices. 


\section{Shigehiko KanaYa}

Shigehiko KanaYa received M.S. and Ph.D. degrees in engineering from Toyohashi University of Technology Japan, in 1987 and 1990 respectively. He was an assistant professor (1990-1999), an associate professor (1999-2001) at Yamagata University, an associate professor at Nara Institute of

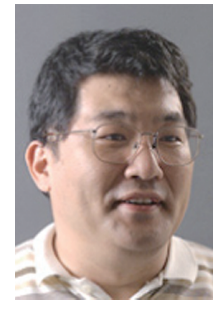
Science and Technology (2001-2004). He is currently professor of graduate school of information science, Nara Institute of Science and Technology since 2004. His research interests include bioinformatics, chemoinformatics and biomedical engineering. 